\title{
Incidence of Cardiotoxicity and Validation of the Heart Failure Association- International Cardio-Oncology Society risk stratification Tool in Patients Treated with Trastuzumab for HER2-Positive Early Breast Cancer.
}

Nicolò Matteo Luca Battisti ( $\sim$ nicolo.battisti@gmail.com )

Royal Marsden NHS Foundation Trust https://orcid.org/0000-0002-1063-1717

Maria Sol Andres

Royal Brompton and Harefield NHS Trust: Royal Brompton and Harefield NHS Foundation Trust

Karla A Lee

Royal Marsden Hospital NHS Trust: Royal Marsden NHS Foundation Trust

Tharshini Ramalingam

Royal Brompton and Harefield NHS Trust: Royal Brompton and Harefield NHS Foundation Trust

Tamsin Nash

Royal Marsden Hospital NHS Trust: Royal Marsden NHS Foundation Trust

Stephanie Mappouridou

Royal Marsden Hospital NHS Trust: Royal Marsden NHS Foundation Trust Nishanthi Senthivel

Royal Marsden Hospital NHS Trust: Royal Marsden NHS Foundation Trust

Kalaprapa Asavisanu

Royal Marsden Hospital NHS Trust: Royal Marsden NHS Foundation Trust

Mariam Obeid

Royal Marsden Hospital NHS Trust: Royal Marsden NHS Foundation Trust

Elli-Sophia Tripodaki

Royal Marsden Hospital NHS Trust: Royal Marsden NHS Foundation Trust

Vasileios Angelis

Royal Marsden Hospital NHS Trust: Royal Marsden NHS Foundation Trust

Emily Fleming

Royal Marsden Hospital NHS Trust: Royal Marsden NHS Foundation Trust

Emily F Goode

Royal Marsden Hospital NHS Trust: Royal Marsden NHS Foundation Trust

Susan John

Royal Marsden Hospital NHS Trust: Royal Marsden NHS Foundation Trust

Stuart D Rosen

Royal Brompton and Harefield NHS Trust: Royal Brompton and Harefield NHS Foundation Trust

Mark Allen

Royal Marsden Hospital NHS Trust: Royal Marsden NHS Foundation Trust

Susannah Stanway

Royal Marsden Hospital NHS Trust: Royal Marsden NHS Foundation Trust

Alexander R Lyon

Royal Brompton and Harefield NHS Trust: Royal Brompton and Harefield NHS Foundation Trust

Alistair Ring

Royal Marsden Hospital NHS Trust: Royal Marsden NHS Foundation Trust

Research Article

Keywords: Breast cancer, trastuzumab, cardiotoxicity, early stage

Posted Date: February 24th, 2021

DOI: https://doi.org/10.21203/rs.3.rs-251663/v1

License: (c) (1) This work is licensed under a Creative Commons Attribution 4.0 International License. Read Full License 
Version of Record: A version of this preprint was published at Breast Cancer Research and Treatment on April 5th, 2021. See the published version at https://doi.org/10.1007/s10549-021-06192-w. 


\section{Abstract}

Purpose

Trastuzumab improves survival in patients with HER2+ early breast cancer. However, cardiotoxicity remains a concern, particularly in the curative setting, and there are limited data on its incidence outside of clinical trials. We retrospectively evaluated the cardiotoxicity rates (left ventricular ejection fraction [LVEF] decline, congestive heart failure [CHF], cardiac death or trastuzumab discontinuation) and assessed the performance of a proposed model to predict cardiotoxicity in routine clinical practice.

\section{Methods}

Patients receiving curative trastuzumab between 2011-2018 were identified. Demographics, treatments, assessments and toxicities were recorded. Fisher's exact test, chi-squared and logistic regression were used.

\section{Results}

931 patients were included in the analysis. Median age was 54 years (range 24-83) and Charlson comorbidity index 0 (0-6), with 195 patients (20.9\%) aged 65 or older. 228 (24.5\%) were smokers. Anthracyclines were given in 608 (65.3\%). Median number of trastuzumab doses was 18 (1-18). The HFA-ICOS cardiovascular risk was low in 401 patients (43.1\%), medium in 454 (48.8\%), high in 70 (7.5\%) and very high in $6(0.6 \%)$.

Overall, 155 (16.6\%) patients experienced cardiotoxicity: LVEF decline $\geq 10 \%$ in 141 (15.1\%), falling below $50 \%$ in 55 (5.9\%), CHF NYHA class II in 42 (4.5\%) and class III-IV in $5(0.5 \%)$ and discontinuation due to cardiac reasons in 35 (3.8\%). No deaths were observed.

Cardiotoxicity rates increased with HFA-ICOS score (14.0\% low, $16.7 \%$ medium, $30.3 \%$ high/very high; $\mathrm{p}=0.002)$.

\section{Conclusions}

Cardiotoxicity was relatively common (16.6\%), but symptomatic heart failure on trastuzumab was rare in our cohort. The HFA-ICOS score identifies patients at high risk of cardiotoxicity

\section{Introduction}

Trastuzumab is a monoclonal antibody targeting the human epidermal growth factor receptor 2 (HER2) and is the standard of care for the management of early-stage and advanced HER2-positive breast cancer.(1) However, treatment with HER2-directed agents is associated with a risk of cardiotoxicity. This most frequently involves an asymptomatic decrease in the left ventricular ejection fraction (LVEF) detected during surveillance before presentation with symptomatic heart failure. Less frequently, rapid development of congestive heart failure (CHF) despite surveillance may develop.(2, 3) Cardiotoxicity associated with anti-HER2 agents is usually reversible with cessation of trastuzumab treatment and cardiac medication, but this may compromise optimal breast cancer treatment.(4) Factors associated with a higher risk of cardiotoxicity in patients receiving trastuzumab include older age, previous or concurrent anthracycline use, pre-existing cardiac dysfunction, pre-existing significant cardiovascular (CV) disease, high body mass index (BMI), antihypertensive therapy and, in older patients, diabetes mellitus.(5-11)

A metanalysis of adjuvant trials reported a risk of advanced heart failure (New York Heart Association (NYHA) class III-IV) of 0.4-2.5\% in patients receiving trastuzumab.(12) Even when anthracyclines are not given, a trial investigating the use of trastuzumab along with taxane-based chemotherapy showed an incidence of cardiotoxicity of $3 \%$ although this was severe only in $0.5 \%$ of trial participants.(13) In contrast, previous real-world experiences have reported a rate of cardiovascular complications in $10-15 \%$ of patients receiving this agent in the curative setting.(14)

Age is a predictor of impaired cardiac function with trastuzumab treatment. This is a concern due to the higher burden of comorbidities and increased risk of adverse outcomes in older individuals.(15) Nonetheless, trastuzumab improves survival and reduces risk of recurrence and is otherwise well tolerated in older patients. The rate of cardiac events in a systematic review of randomized studies including data on patients aged over 60 years was $5 \%$.(16) However, the incidence is unclear outside of clinical trials, which tend to recruit patients who are younger, with normal baseline cardiac function and who have a lower burden of co-morbidities including pre-existing CV disease.

Therefore, predicting the cardiotoxicity of anti-HER2 agents is of considerable importance. Cardiac risk scores have been developed based on prospective trial(12) and retrospective registry data.(14) However, independent validation is needed before they can be considered for general use. The Heart Failure Association (HFA) of the European Society of Cardiology (ESC) together with the International Cardio-Oncology Society (ICOS) have recently developed a risk stratification tool (HFA-ICOS Risk Tool) to evaluate the likelihood of cardiotoxicity at baseline for patients receiving HER2-directed treatments.(17) In this study we investigated the rates of cardiotoxicity secondary to trastuzumab for early-stage HER2-positive breast cancer in a breast cancer service, comparing rates in older versus younger patients, and assessed the performance of HFA-ICOS cardiovascular risk prediction tool in this population.

\section{Methods}

This analysis is a retrospective study of patients who received trastuzumab for HER2-positive early breast cancer (EBC) between 01/01/2011 and 31/12/2018 at the Royal Marsden Hospital NHS Foundation Trust. Eligible patients had curable disease (TNM stages: T1-4, N0-3, M0) and received trastuzumab in the neoadjuvant or adjuvant setting. Patients who received part of the course of treatment elsewhere or those with advanced-stage breast cancer were not eligible for the analysis. This analysis was approved as a service evaluation (SE842) at the Royal Marsden NHS Foundation Trust. 


\section{Baseline data collection}

Baseline patient characteristics at initiation of trastuzumab were collected and included: date of birth, age at diagnosis, date of last follow-up, date of death, weight, body mass index (BMI), comorbidities, smoking history, obesity, alcohol consumption, concurrent medications, Eastern Cooperative Oncology Group Performance Score (ECOG PS), menopausal status. Specifically, data on CV comorbidities and risk factors were collected and included: diabetes mellitus, hypertension, hypercholesterolemia, coronary artery disease, cerebrovascular disease, peripheral artery disease, heart failure and NYHA classification, rheumatic heart disease, arrhythmias, congenital heart disease, valvular heart disease, cardiomyopathy, aortic aneurysm, thromboembolic disease, pulmonary hypertension, pericardial disease and chronic kidney disease. A non-age adjusted Charlson Comorbidity Index (CCI) was calculated for each patient based on comorbidities at baseline. Specific data on medications relevant to cardiovascular risk were recorded and included: beta-blockers, angiotensin-converting enzyme (ACE) inhibitors, angiotensin receptor blockers, mineralocorticoid receptor blockers, diuretics, digitalis, calcium channel blockers, antiplatelets, anticoagulants and statins. Blood tests results including haemoglobin, white blood count (WBC) and creatinine measurements and LVEF measured on multiple-gated acquisition (MUGA) scan or echocardiogram as per local practice were also recorded at baseline.

Baseline data were collected regarding the primary tumour including: date of diagnosis, histology, grade, ER status and Allred score, PR status and Allred score, HER2 testing method, best stage (i.e., the worst stage between clinical stage and pathological stage), laterality.

Radiotherapy and systemic therapy data were collected. These included use of chemotherapy, anthracyclines, taxanes, platinum compounds, pertuzumab, radiotherapy, endocrine agents, along with setting (adjuvant versus neoadjuvant), cumulative dose of anthracyclines, number of chemotherapy cycles and number of doses of trastuzumab.

The baseline cardiovascular risk of these patients was classified as low/medium/high/very high based on the recommendations of the HFA-ICOS Risk Tool developed for HER2-targeted agents.(17)

\section{Follow-up and outcomes}

Data on LVEF from MUGA scan or echocardiogram performed as per National Cancer Research Institute recommendations in the UK(18) until trastuzumab completion or discontinuation were recorded (i.e., baseline, 16 and 23 weeks for patients receiving taxanes alone and before and after anthracycline use for those receiving sequential chemotherapy regimens). Cardiac adverse outcomes were defined as: death due to cardiac reasons, LVEF decline of $\geq 10 \%$, LVEF decline to below 50\%, congestive heart failure (CHF) (NYHA class II and III-IV) and trastuzumab discontinuation (temporary or permanent) due to cardiac toxicity. Reasons for discontinuing trastuzumab not related to cardiotoxicity and management of cardiac events with specialist referrals and medications were also recorded.

\section{Statistical analysis}

Analyses were performed in Stata/MP 16.0.(19) A p<0.05 was considered statistically significant. Baseline patients and breast cancer characteristics were tabulated and compared among age groups ( $\geq 65$ years and $<65$ years) and HFA-ICOS CV risk groups (low versus medium versus high versus very high) by using chi-squared, Fisher's statistics, two-sample t tests and 3-way ANOVA. Similarly, exposure to anticancer treatments was compared among age and HFAICOS CV risk groups. Baseline LVEF measurements were compared with those at trastuzumab completion in the overall population and according to age group for those patients undergoing a MUGA scan or an echocardiogram at treatment initiation and specifically for those undergoing a baseline echocardiogram.

Cardiac event rates occurring at any time during the course of trastuzumab and subsequent follow-up were estimated and compared according to age ( $\geq 65$ vs $<65$ years) and HFA-ICOS CV risk (low vs medium vs high/very high). These rates were also compared based on menopausal status and use of statins at baseline. Reasons for trastuzumab discontinuation and management of cardiac events were also compared among these patient groups.

Logistical regression was used to calculate the odds of cardiac events based on HFA-ICOS risk category. The performance of the HFA-ICOS Risk Tool to predict cardiotoxicity was evaluated by calculating sensitivity, specificity, positive predictive value (PPV) and negative predictive value (NPV). We also composed receiver-operating characteristic $(\mathrm{ROC})$ curves and calculated the area under the curve for the prediction model.

\section{Results}

\section{Population characteristics}

Between January 2011 and December 2018, 1,094 patients initiated trastuzumab in the curative setting for HER2+ EBC at The Royal Marsden NHS Foundation Trust. The analysis was restricted to 931 patients who completed the entire course of trastuzumab at our Institution for whom cardiac assessments were available (CONSORT diagram [Fig. 1]).

Patient characteristics and tumour characteristics are shown in Table 1. No significant differences in patient and tumour characteristics were observed in those aged $\geq 65$ years compared with their younger counterparts. Comorbidities and CV risk factors are outlined in Table 2 . Patients aged 65 years and older had a higher prevalence of diabetes mellitus, hypertension and hypercholesterolemia compared with the younger patients ( $<65$ years old). At trastuzumab initiation, a higher proportion of patients aged $\geq 65$ years were on cardioprotective medications including beta-blockers, ACE inhibitors, angiotensin receptor blockers and mineralocorticoid receptor blockers (Table 2).

Of the 931 patients, based on the HFA-ICOS risk stratification tool 401 (43.1\%) had a low baseline CV risk, 454 patients (48.8\%) were medium risk, 70 patients (7.5\%) were high risk, and 6 patients $(0.6 \%)$ were very high risk. 
Trastuzumab was given in the adjuvant setting only in 584 patients $(62.7 \%)$, whereas $347(37.3 \%)$ received trastuzumab neoadjuvantly and continued treatment in the adjuvant setting. The median number of doses given was 18 (range 1-18). The majority of patients received a sequential combination of anthracyclines and taxanes (594 [63.8\%]), whilst 288 (30.9\%) received taxanes alone. Pertuzumab was added to trastuzumab in 158 patients (17.0\%) and adjuvant radiotherapy was given to 689 patients (74.0\%). Among 638 patients with ER-positive disease, tamoxifen was initially prescribed for 379 patients (59.4\%) and an aromatase inhibitor for $226(35.4 \%)$.

Table 3 report the treatments given in the overall population and based on age and HFA-ICOS risk category. Anthracyclines were added to a taxane less frequently in older patients ( $\geq 65$ years 68 [34.9\%] versus <65 years 526 [71.5\%]; p 0.001) and in those with increasing HFA-ICOS risk score (low 271 [67.6\%] versus medium 291 [64.1\%] versus high 31 [44.3\%] versus very high 1 [16.7\%]; p 0.001). Similarly, older patients and those with higher CV risk were more likely to receive trastuzumab only in the adjuvant setting rather than in the neoadjuvant setting.

LVEF at baseline and upon trastuzumab completion in the overall population and according to age group are reported in Fig. 2.

\section{Cardiac events and their management}

Cardiac adverse events occurred in 155 patients (16.6\%) (Table 4 and Fig. 3). No cardiac deaths were observed in this cohort. One hundred and forty-one patients (15.1\%) experienced a LVEF decline $\geq 10 \%$ and 55 (5.91\%) below $50 \%$. Forty-seven patients (5.0\%) developed symptomatic heart failure. In this cohort, 42 patients (4.5\%) had mild symptoms (NYHA class II) and 5 patients (0.5\%) had more severe symptomatic heart failure (NYHA class III-IV).

Trastuzumab was discontinued due to cardiotoxicity in 35 patients (3.76\%). No significant differences in cardiotoxicity were seen according to age group.

Table 5 outlines the management of cardiotoxicity events. One hundred and seventeen patients (12.6\%) required a referral to a cardiologist provided by a specialist cardio-oncology service. Beta-blockers (preferably carvedilol) were prescribed in 57 patients (6.1\%), ACE inhibitors or angiotensin receptor blockers in 99 (10.6\%), mineralocorticoid receptor blockers (eplerenone) in 5 patients $(0.54 \%)$, diuretics in 16 patients $(1.7 \%)$ and statins were started in 17 patients (1.8\%) either by the treating oncologist or by the cardiologist. No significant differences were observed in the management of cardiac events based on age. The use of cardioprotective medications following this specific toxicity increased with increasing HFA-ICOS risk category.

\section{Performance of the HFA-ICOS risk prediction model}

Increasing CV risk based on the HFA-ICOS category correlated with increasing rates of cardiac events on trastuzumab: the overall rates of cardiotoxicity was $14.0 \%$ in patients classified as low risk versus $16.7 \%$ with medium risk versus $30.3 \%$ classified as baseline as high or very high risk ( $p=0.002)$ (Fig. 4 ).

The HFA-ICOS score also correlated with increasing rates of cardiac toxicity: $7.6 \%$ for low-risk patients with a score of 0 ( $n=66$ ); $15.2 \%$ for low-risk patients with a score of $1(n=335) ; 16.0 \%$ for medium-risk patients with a score of $2(n=263) ; 18.3 \%$ for medium-risk patients with a score of 3 ( $n=120) ; 16.9 \%$ for mediumrisk patients with a score of $4(n=71) ; 30.3 \%$ for high- to very high-risk patients with a score $\geq 5(n=76)(p=0.0147)($ Fig. 5).

The HFA-ICOS Risk Tool had a sensitivity of $14.8 \%$, a specificity of $93.2 \%$, a PPV of $30.3 \%$ and a NPV of $84.6 \%$ when predicting any cardiac event on trastuzumab in patients classified as low/medium risk versus those classified as high/very high risk. Area under the ROC curve for the predictive model for any cardiac toxicity was 0.56 .

\section{Discussion}

This is a large retrospective single centre study analysing cardiotoxicity incidence and outcomes for patients receiving trastuzumab for curable HER2-positive breast cancer, with a particular focus on outcomes for the older age group and according to baseline HFA-ICOS Risk. A significant proportion of these patients (43.1\%) had a low cardiovascular risk profile based on the HFA-ICOS assessment tool. Nonetheless, more than a half had medium, high or very high risk and establishing the rates of cardiotoxicity in the real world is crucial especially in the curative setting.

A key result of our analysis is that the incidence of clinically serious symptomatic heart failure in patients receiving curative trastuzumab outside clinical trials is low (5.0\%), with no fatal cardiotoxicity, although various degrees of cardiac toxicity may occur in up to $16.6 \%$ of patients on this treatment. These results are comparable to a recent pooled analysis of the trastuzumab registration trials which showed a small to modest risk of cardiotoxicity ranging between $5.5 \%$ and 19.4\%.(20) The importance of this analysis is that it includes a real-world population of patients not enrolled in clinic trials and therefore may be particularly useful to inform routine clinical practice.

Benchmarking the incidence of cardiac events for patients receiving trastuzumab in the curative setting is also important in the context of the studies investigating de-escalation strategies. In our series one third of patients received taxanes alone and in a similar population with node-negative EBC, the APT study reported even lower rates of cardiac toxicity, with $0.5 \%$ of patients experiencing grade 3 left ventricular systolic dysfunction and $3 \%$ reporting asymptomatic LVEF decline.(13) In our series only $3.8 \%$ of patients did not complete a full one-year course of trastuzumab due to cardiac toxicity. The PERSEPHONE study suggested non-inferior efficacy of 6 months of treatment compared with 12 months along with a substantial reduction in cardiac events from $12 \%$ to $9 \% .(21)$

This study suggests that there are no differences in the rates of cardiac adverse events according to age. This is consistent with previous analyses showing that most patients aged $\geq 66$ years are able to complete a one-year course of trastuzumab without complications,(22) although comorbidities remain critical in determining the risk of cardiotoxicity.(23) One variable that may explain the lack of effect of age alone is the rate of anthracycline chemotherapy which was 
significantly lower in the patients $\geq 65$ years (34.9\%) versus the younger patients $<65$ years $(71.5 \%)$. Therefore, the increased risk portended by increasing age may be balanced by the higher anthracycline chemotherapy use in the younger patients.

Our analysis also included a substantial proportion of patients with medium/high cardiovascular risk (56.9\%). The registration trials of trastuzumab mandated stringent cardiac monitoring, limited the cumulative dose of anthracyclines to $300 \mathrm{mg} / \mathrm{m}^{2}$ and excluded subjects with abnormal baseline cardiac function. This consideration makes real-world experiences useful since the risk of cardiac toxicity on trastuzumab varies according to the use of previous chemotherapy, pre-existing heart disease and cardiovascular risk factors.(24) Therefore, identifying the baseline cardiovascular risk and developing prediction models able to identify those patients at higher risk of experiencing cardiac events remains particularly valuable.(17)

The HFA-ICOS risk score had a good correlation with the incidence of cardiotoxicity in our analysis, with $30.3 \%$ of patients with a high- to very high-risk score experiencing any cardiac event compared with $16.7 \%$ of those with medium risk and $14.0 \%$ of those with low risk. We documented a similar pattern also for specific types of cardiac adverse events, including LVEF decline, CHF and trastuzumab discontinuations. Importantly, the HFA-ICOS score had a high NPV $(86.0 \%)$ which is highly desirable to identify those patients who are not at risk of cardiac toxicity in this setting. It was less sensitive to discriminate between the low and medium risk cohorts with similar event rates and did not identify the cohort at absolute low risk $(<5 \%)$.

This study has a number of limitations. At our institution, the measurement of cardiac biomarkers such as troponin and natriuretic peptides is not routine practice; therefore, despite their desirability where available,(17) they have not been included in the model. In this series, baseline cardiac assessments involved either MUGA scans or echocardiograms to measure LVEF which may have introduced bias. Measuring the global longitudinal strain (GLS) using speckle tracking echocardiography has become standard practice in our hospital only since 2016 and therefore this parameter has not been captured in our cohort. GLS has recently emerged as a new marker of subclinical ventricular dysfunction demonstrating a stronger association with prognosis compared with LVEF in patients with cardiac conditions not related to cancer.(25) Various observational studies suggested its potential role accurately to predict the cardiotoxicity of anticancer agents and guide cardioprotective treatment.(26, 27) Our analysis is retrospective and therefore may be subject to selection bias as we included patients who were deemed fit to receive trastuzumab. Finally, excluding patients who did not receive a full course of trastuzumab at our institution may have also contributed to selection bias.

This analysis has some major strengths as well. We have demonstrated within a large cohort that overall rates of serious cardiotoxicity associated with trastuzumab are low, but absolute rate of all cardiotoxicity is clinically significant (16.6\%), and dependent on the individual cardiovascular risk profile at baseline. Our study provides evidence that rates of cardiotoxicity on trastuzumab do not differ based on age in a real-world population. Furthermore, we have included patients receiving contemporary chemotherapy and targeted treatment regimens which make our findings applicable to current practice. Our study fills a gap of knowledge by providing evidence of external validation of a prediction model of cardiac toxicity in a population receiving treatment with substantial chances of cure.(1) This aspect is particularly valuable in the older patient population where competing risks of morbidity and mortality are more relevant.

These data should be considered when discussing risks and benefits of trastuzumab in older patients with HER2-positive EBC and prospective validation of the use of the HFA-ICOS Risk Tool is warranted.

\section{Declarations}

\section{Acknowledgements}

The authors wish to acknowledge the support of the Royal Marsden NIHR Biomedical Research Centre for Cancer and The Cridlan Ross Smith Charitable Trust. ARL is supported by the Leducq Transatlantic Cardio-Oncology Network.

\section{Funding}

Dr Battisti is supported by a fellowship of The Cridlan Ross Smith Charitable Trust. Dr Battisti and Dr Ring wish to acknowledge the support of The Royal Marsden NIHR Biomedical Research Centre for Cancer. Dr Tripodaki is funded by a scholarship from the Hellenic Society of Clinical Oncology (HESMO). The remaining authors have no funding to report.

\section{Conflicts of interest/Competing interests}

Dr Battisti has received travel grants from Genomic Health and Pfizer and speaker fees from Pfizer and AbbVie. Dr Lyon has received speaker, advisory board or consultancy fees and/or research grants from Pfizer, Novartis, Servier, Astra Zeneca, Bristol Myers Squibb, GSK, Amgen, Takeda, Roche, Janssens-Cilag Ltd, Clinigen Group, Eli Lily, Eisai Ltd, Ferring Pharmaceuticals, Boehringer Ingelheim, Akcea Therapeutics, Myocardial Solutions, iOWNA Health and Heartfelt Technologies Ltd. Dr Ring has received advisory board and speaker fees from Roche, Novartis, Pfizer, MSD and Lilly. Dr Andres, Dr Lee, Dr Ramalingam, Dr Nash, Dr Mappouridou, Dr Senthivel, Dr Asavisanu, Dr Obeid, Dr Tripodaki, Dr Angelis, Dr Fleming, Dr Goode, Dr John, Professor Rosen, Dr Allen, Dr Stanway have no conflicts of interest.

\section{Ethics approval}

This analysis was approved as a Service Evaluation by The Committee for Clinical Review of The Royal Marsden NHS Foundation Trust.

\section{Consent to participate (include appropriate statements)}

Not applicable. 


\section{Consent for publication}

No individual person's data are included in the manuscript in any form and therefore no consents for publication were required.

\section{Availability of data and material}

The datasets generated during and/or analysed during the current study are available from the corresponding author on reasonable request.

\section{Code availability (software application or custom code)}

The statistical analysis code generated during the current study is available from the corresponding author on reasonable request.

\section{Authors' contributions}

NMLB, MA, ARL and AR conceived and designed the analysis. NMLB, KAL, TN, SM, NS, KA, MO, EST, VA, EF, EFG, SJ collected the data. NMLB performed the analysis. NMLB, MSA, KAL, TR, TN, SM, NS, KA, MO, EST, VA, EF, EFG, SJ, SDR, MA, SS, ARL and AR wrote the paper.

\section{References}

1. Hayes DF (2019) HER2 and Breast Cancer - A Phenomenal Success Story. N Engl J Med 381(13):1284-1286

2. Perez EA, Rodeheffer R (2004) Clinical cardiac tolerability of trastuzumab. J Clin Oncol 22(2):322-329

3. Keefe DL (2002) Trastuzumab-associated cardiotoxicity. Cancer 95(7):1592-1600

4. Eiger D, Pondé NF, Agbor-Tarh D, Moreno-Aspitia A, Piccart M, Hilbers FS et al (2020) Long-term cardiac outcomes of patients with HER2-positive breast cancer treated in the adjuvant lapatinib and/or trastuzumab Treatment Optimization Trial. Br J Cancer 122(10):1453-1460

5. Bowles EJ, Wellman R, Feigelson HS, Onitilo AA, Freedman AN, Delate T et al (2012) Risk of heart failure in breast cancer patients after anthracycline and trastuzumab treatment: a retrospective cohort study. J Natl Cancer Inst 104(17):1293-1305

6. Ewer SM, Ewer MS (2008) Cardiotoxicity profile of trastuzumab. Drug safety 31(6):459-467

7. Guenancia C, Lefebvre A, Cardinale D, Yu AF, Ladoire S, Ghiringhelli F et al (2016) Obesity As a Risk Factor for Anthracyclines and Trastuzumab Cardiotoxicity in Breast Cancer: A Systematic Review and Meta-Analysis. J Clin Oncol 34(26):3157-3165

8. Romond EH, Jeong JH, Rastogi P, Swain SM, Geyer CE Jr, Ewer MS et al (2012) Seven-year follow-up assessment of cardiac function in NSABP B-31, a randomized trial comparing doxorubicin and cyclophosphamide followed by paclitaxel (ACP) with ACP plus trastuzumab as adjuvant therapy for patients with node-positive, human epidermal growth factor receptor 2-positive breast cancer. J Clin Oncol 30(31):3792-3799

9. Russell SD, Blackwell KL, Lawrence J, Pippen JE Jr, Roe MT, Wood F et al (2010) Independent adjudication of symptomatic heart failure with the use of doxorubicin and cyclophosphamide followed by trastuzumab adjuvant therapy: a combined review of cardiac data from the National Surgical Adjuvant breast and Bowel Project B-31 and the North Central Cancer Treatment Group N9831 clinical trials. J Clin Oncol 28(21):3416-3421

10. Slamon DJ, Leyland-Jones B, Shak S, Fuchs H, Paton V, Bajamonde A et al (2001) Use of chemotherapy plus a monoclonal antibody against HER2 for metastatic breast cancer that overexpresses HER2. N Engl J Med 344(11):783-792

11. Suter TM, Procter M, van Veldhuisen DJ, Muscholl M, Bergh J, Carlomagno C et al (2007) Trastuzumab-associated cardiac adverse effects in the herceptin adjuvant trial. J Clin Oncol 25(25):3859-3865

12. Moja L, Tagliabue L, Balduzzi S, Parmelli E, Pistotti V, Guarneri V et al (2012) Trastuzumab containing regimens for early breast cancer. Cochrane Database Syst Rev 2012(4):Cd006243

13. Dang C, Guo H, Najita J, Yardley D, Marcom K, Albain K et al (2016) Cardiac Outcomes of Patients Receiving Adjuvant Weekly Paclitaxel and Trastuzumab for Node-Negative, ERBB2-Positive Breast Cancer. JAMA Oncol 2(1):29-36

14. Tarantini L, Cioffi G, Gori S, Tuccia F, Boccardi L, Bovelli D et al (2012) Trastuzumab adjuvant chemotherapy and cardiotoxicity in real-world women with breast cancer. J Card Fail 18(2):113-119

15. Reeder-Hayes KE, Meyer AM, Hinton SP, Meng K, Carey LA, Dusetzina SB (2017) Comparative Toxicity and Effectiveness of Trastuzumab-Based Chemotherapy Regimens in Older Women With Early-Stage Breast Cancer. J Clin Oncol 35(29):3298-3305

16. Brollo J, Curigliano G, Disalvatore D, Marrone BF, Criscitiello C, Bagnardi V et al (2013) Adjuvant trastuzumab in elderly with HER-2 positive breast cancer: a systematic review of randomized controlled trials. Cancer Treat Rev 39(1):44-50

17. Lyon AR, Dent S, Stanway S, Earl H, Brezden-Masley C, Cohen-Solal A et al. Baseline cardiovascular risk assessment in cancer patients scheduled to receive cardiotoxic cancer therapies: a position statement and new risk assessment tools from the Cardio-Oncology Study Group of the Heart Failure Association of the European Society of Cardiology in collaboration with the International Cardio-Oncology Society. Eur J Heart Fail. 2020

18. Jones AL, Barlow M, Barrett-Lee PJ, Canney PA, Gilmour IM, Robb SD et al (2009) Management of cardiac health in trastuzumab-treated patients with breast cancer: updated United Kingdom National Cancer Research Institute recommendations for monitoring. Br J Cancer 100(5):684-692

19. StataCorp. Stata Statistical Software: Release 16. 2019

20. de Azambuja E, Ponde N, Procter M, Rastogi P, Cecchini RS, Lambertini M et al (2020) A pooled analysis of the cardiac events in the trastuzumab adjuvant trials. Breast Cancer Res Treat 179(1):161-171

21. Earl HM, Hiller L, Vallier AL, Loi S, McAdam K, Hughes-Davies L et al (2019) 6 versus 12 months of adjuvant trastuzumab for HER2-positive early breast cancer (PERSEPHONE): 4-year disease-free survival results of a randomised phase 3 non-inferiority trial. Lancet 393(10191):2599-2612 
22. Vaz-Luis I, Keating NL, Lin NU, Lii H, Winer EP, Freedman RA (2014) Duration and toxicity of adjuvant trastuzumab in older patients with early-stage breast cancer: a population-based study. J Clin Oncol 32(9):927-934

23. Serrano C, Cortés J, De Mattos-Arruda L, Bellet M, Gómez P, Saura C et al (2012) Trastuzumab-related cardiotoxicity in the elderly: a role for cardiovascular risk factors. Ann Oncol 23(4):897-902

24. Henry ML, Niu J, Zhang N, Giordano SH, Chavez-MacGregor M (2018) Cardiotoxicity and Cardiac Monitoring Among Chemotherapy-Treated Breast Cancer Patients. JACC Cardiovasc Imaging 11(8):1084-1093

25. Laufer-Perl M, Gilon D, Kapusta L, lakobishvili Z. The Role of Speckle Strain Echocardiography in the Diagnosis of Early Subclinical Cardiac Injury in Cancer Patients-Is There More Than Just Left Ventricle Global Longitudinal Strain? J Clin Med. 2021;10(1)

26. Čelutkienè J, Pudil R, López-Fernández T, Grapsa J, Nihoyannopoulos P, Bergler-Klein J et al. Role of cardiovascular imaging in cancer patients receiving cardiotoxic therapies: a position statement on behalf of the Heart Failure Association (HFA), the European Association of Cardiovascular Imaging (EACVI) and the Cardio-Oncology Council of the European Society of Cardiology (ESC). Eur J Heart Fail. 2020

27. Thavendiranathan P, Negishi T, Somerset E, Negishi K, Penicka M, Lemieux J et al (2021) Strain-Guided Management of Potentially Cardiotoxic Cancer Therapy. J Am Coll Cardiol 77(4):392-401

\section{Tables}


Table 1

Patient and tumour characteristics at baseline in the overall population and according to age group.

\begin{tabular}{|c|c|c|c|c|c|c|c|c|}
\hline \multirow[t]{2}{*}{ Characteristics } & & \multirow{2}{*}{\multicolumn{2}{|c|}{$\begin{array}{l}\text { Overall } \\
N=931\end{array}$}} & \multicolumn{4}{|c|}{ Age group } & \multirow[t]{2}{*}{$p$ value } \\
\hline & & & & \multicolumn{2}{|c|}{$\begin{array}{l}<65 \text { years } \\
N=736\end{array}$} & \multicolumn{2}{|c|}{$\begin{array}{l}\geq 65 \text { years } \\
N=195\end{array}$} & \\
\hline \multicolumn{9}{|l|}{ Continuous variables } \\
\hline \multirow[t]{5}{*}{ Age (years) } & Median & \multicolumn{2}{|l|}{54} & \multicolumn{2}{|l|}{50} & \multicolumn{2}{|l|}{69} & \multirow[t]{5}{*}{-} \\
\hline & IQR & \multicolumn{2}{|c|}{$46-63$} & \multicolumn{2}{|c|}{$43-56$} & \multicolumn{2}{|c|}{$67-73$} & \\
\hline & Mean & \multicolumn{2}{|l|}{54.3} & \multicolumn{2}{|l|}{50.0} & \multicolumn{2}{|c|}{70.9} & \\
\hline & Standard deviation & \multicolumn{2}{|l|}{11.9} & \multicolumn{2}{|l|}{9.0} & \multicolumn{2}{|l|}{4.6} & \\
\hline & Range & \multicolumn{2}{|c|}{$24-83$} & \multicolumn{2}{|c|}{$24-64$} & \multicolumn{2}{|c|}{$65-83$} & \\
\hline Weight $(\mathrm{kg})^{*}$ & Median & 69 & & 69.0 & & 68.8 & & 0.555 \\
\hline & IQR & 60.8 & 78.9 & 60.6 & 79.0 & 61.5 & 77.7 & \\
\hline & Mean & 71.0 & & 71.3 & & 70.1 & & \\
\hline & Standard deviation & 14.8 & & 15.4 & & 12.4 & & \\
\hline & Range & 42.5 & 140.0 & 42.5 & 140.0 & 43.7 & 06.6 & \\
\hline $\mathrm{BMI}\left(\mathrm{kg} / \mathrm{m}^{2}\right)^{\wedge}$ & Median & 25.4 & & 25.4 & & 26.7 & & 0.073 \\
\hline & IQR & 22.7 & 30.0 & 22.0 & 30.0 & 23.8 & 30.2 & \\
\hline & Mean & 26.8 & & 26.7 & & 27.2 & & \\
\hline & Standard deviation & 5.50 & & 5.70 & & 4.7 & & \\
\hline & Range & 15.9 & 51.8 & 15.9 & 51.8 & 17.3 & 42.2 & \\
\hline Charlson comorbidity index & Median & 0 & & 0 & & 0 & & 0.259 \\
\hline & IQR & $0-2$ & & $0-0$ & & $0-1$ & & \\
\hline & Mean & 0.9 & & 0.9 & & 1.0 & & \\
\hline & Standard deviation & 1.1 & & 1.0 & & 1.1 & & \\
\hline & Range & $0-6$ & & $0-5$ & & $0-6$ & & \\
\hline Categorical variables & & $\mathrm{N}$ & $\%$ & $\mathrm{~N}$ & $\%$ & $\mathrm{~N}$ & $\%$ & \\
\hline Sex & Female & 930 & 99.9 & 736 & 100.00 & 194 & 99.5 & - \\
\hline & Male & 1 & 0.1 & 0 & 0.00 & 1 & 0.5 & - \\
\hline ECOG PS & 0 & 826 & 88.7 & 679 & 92.3 & 147 & 75.4 & 0.001 \\
\hline & 1 & 102 & 11.0 & 57 & 7.7 & 45 & 23.1 & 0.001 \\
\hline & 2 & 3 & 0.3 & 0 & 0.0 & 3 & 1.5 & 0.009 \\
\hline Menopausal status & Pre/perimenopausal & 427 & 45.9 & 427 & 58.0 & 0 & 0.0 & - \\
\hline & Postmenopausal & 504 & 54.1 & 309 & 42.0 & 195 & 100.0 & 0.001 \\
\hline Status (on $13 / 05 / 2020$ ) & Dead & 51 & 5.5 & 36 & 4.9 & 15 & 7.7 & 0.155 \\
\hline & Alive & 880 & 94.5 & 700 & 95.1 & 180 & 92.3 & - \\
\hline Previous (remote) use of che & notherapy & 45 & 4.8 & 35 & 4.8 & 10 & 5.1 & 0.851 \\
\hline Previous (remote) use of ant & racyclines & 29 & 3.1 & 23 & 3.1 & 6 & 3.1 & 0.999 \\
\hline Previous (remote) use of tras & uzumab & 9 & 1.0 & 9 & 1.2 & 0 & 0.0 & 0.217 \\
\hline Histology & Ductal & 885 & 95.1 & 706 & 95.9 & 179 & 91.8 & 0.022 \\
\hline & Lobular & 38 & 4.1 & 25 & 3.4 & 13 & 6.7 & 0.064 \\
\hline & Mixed ductal/lobular & 5 & 0.5 & 3 & 0.4 & 2 & 1.0 & 0.282 \\
\hline & Other & 2 & 0.2 & 1 & 0.1 & 1 & 0.5 & 0.376 \\
\hline & Missing & 1 & 0.1 & 1 & 0.1 & 0 & 0.0 & - \\
\hline
\end{tabular}




\begin{tabular}{|c|c|c|c|c|c|c|c|c|}
\hline \multicolumn{2}{|l|}{ Characteristics } & \multirow{3}{*}{\multicolumn{2}{|c|}{$\begin{array}{l}\text { Overall } \\
N=931\end{array}$}} & \multicolumn{4}{|c|}{ Age group } & \multirow{4}{*}{$\begin{array}{l}\text { p value } \\
0.999\end{array}$} \\
\hline & & & & \multirow{2}{*}{\multicolumn{2}{|c|}{$\begin{array}{l}<65 \text { years } \\
N=736\end{array}$}} & \multirow{2}{*}{\multicolumn{2}{|c|}{$\begin{array}{l}\geq 65 \text { years } \\
N=195\end{array}$}} & \\
\hline & & & & & & & & \\
\hline \multirow[t]{4}{*}{ Grade } & 1 & 15 & 1.6 & 12 & 1.6 & 3 & 1.5 & \\
\hline & 2 & 332 & 35.7 & 263 & 35.7 & 69 & 35.4 & 0.867 \\
\hline & 3 & 570 & 61.2 & 448 & 60.9 & 122 & 62.6 & 0.868 \\
\hline & Missing & 14 & 1.5 & 13 & 1.8 & 1 & 0.5 & - \\
\hline \multirow[t]{2}{*}{ ER status } & Negative & 293 & 31.5 & 226 & 30.7 & 67 & 34.4 & 0.341 \\
\hline & Positive & 638 & 68.5 & 510 & 69.3 & 128 & 65.6 & 0.341 \\
\hline \multirow[t]{3}{*}{ PgR status } & Negative & 447 & 48.0 & 340 & 46.2 & 107 & 54.9 & 0.017 \\
\hline & Positive & 452 & 48.5 & 373 & 50.7 & 79 & 40.5 & 0.017 \\
\hline & Missing & 32 & 3.4 & 23 & 3.1 & 9 & 4.6 & - \\
\hline \multirow[t]{3}{*}{ HER2 testing method } & $\mathrm{IHC}$ & 611 & 65.6 & 494 & 67.1 & 117 & 60.0 & - \\
\hline & ISH & 201 & 21.6 & 146 & 19.9 & 55 & 28.2 & - \\
\hline & Unknown & 119 & 12.8 & 96 & 13.0 & 23 & 11.8 & - \\
\hline \multirow[t]{4}{*}{ Best stage ${ }^{\sim}$} & I & 212 & 22.8 & 163 & 22.1 & 49 & 25.1 & 0.386 \\
\hline & ॥ & 551 & 59.2 & 442 & 60.0 & 109 & 55.9 & 0.324 \\
\hline & III & 162 & 17.4 & 127 & 17.3 & 35 & 17.9 & 0.831 \\
\hline & Missing & 6 & 0.6 & 4 & 0.5 & 2 & 1.0 & - \\
\hline \multirow[t]{3}{*}{ Laterality } & Right & 450 & 48.3 & 355 & 48.2 & 95 & 48.7 & 0.936 \\
\hline & Left & 467 & 50.2 & 370 & 50.3 & 97 & 49.7 & 0.936 \\
\hline & Bilateral $\infty$ & 14 & 1.5 & 11 & 1.5 & 3 & 1.5 & 0.999 \\
\hline
\end{tabular}


Table 2

Comorbidities, cardiovascular risk factors and concurrent medications at baseline in the overall population and according to age group.

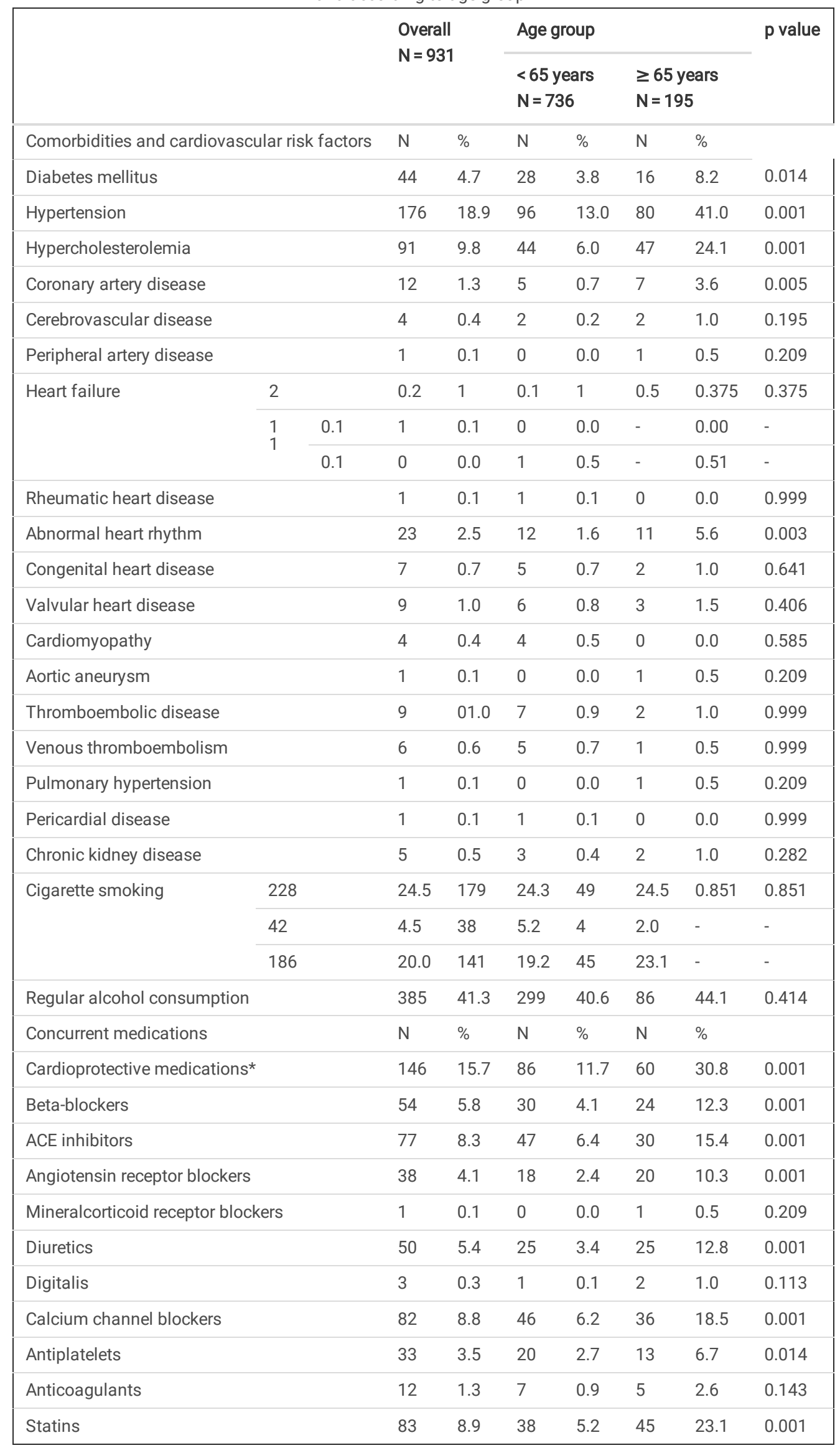


Table 3

Treatment characteristics and exposure in the overall population and according to age and HFA/ICOS risk group.

\begin{tabular}{|c|c|c|c|c|c|c|c|c|c|c|c|c|c|c|c|c|c|}
\hline & & \multirow{2}{*}{\multicolumn{2}{|c|}{$\begin{array}{l}\text { Overall } \\
N=931\end{array}$}} & \multicolumn{4}{|c|}{ Age group } & \multirow{2}{*}{$\begin{array}{l}p \\
\text { value }\end{array}$} & \multicolumn{7}{|c|}{ HFA-ICOS risk group } & \multirow{2}{*}{\multicolumn{2}{|c|}{$p$ value }} \\
\hline & & & & \multicolumn{2}{|c|}{$\begin{array}{l}<65 \text { years } \\
N=736\end{array}$} & \multicolumn{2}{|c|}{$\begin{array}{l}\geq 65 \text { years } \\
N=195\end{array}$} & & \multicolumn{2}{|c|}{$\begin{array}{l}\text { Low } \\
N=401\end{array}$} & \multicolumn{2}{|c|}{$\begin{array}{l}\text { Medium } \\
\mathrm{N}=454\end{array}$} & \multicolumn{2}{|c|}{$\begin{array}{l}\text { High } \\
N=70\end{array}$} & $\begin{array}{l}\text { Very } \\
\text { high } \\
N=6\end{array}$ & & \\
\hline $\begin{array}{l}\text { Treatment } \\
\text { characteristics }\end{array}$ & Category & $\mathrm{N}$ & $\%$ & $\mathrm{~N}$ & $\%$ & $\mathrm{~N}$ & $\%$ & $\mathrm{~N}$ & $\%$ & $\mathrm{~N}$ & $\%$ & $\mathrm{~N}$ & $\%$ & $\mathrm{~N}$ & $\%$ & & \\
\hline \multirow[t]{6}{*}{$\begin{array}{l}\text { Concurrent } \\
\text { chemotherapy }\end{array}$} & $\begin{array}{l}\text { No } \\
\text { chemotherapy }\end{array}$ & 10 & 1.1 & 7 & 0.9 & 3 & 1.5 & 0.445 & 3 & 0.7 & 6 & 1.3 & 0 & 0.0 & 1 & 16.7 & 0.002 \\
\hline & $\begin{array}{l}\text { Anthracycline } \\
+ \text { taxanes }\end{array}$ & 594 & 63.8 & 526 & 71.5 & 68 & 34.9 & 0.001 & 271 & 67.6 & 291 & 64.1 & 31 & 44.3 & 1 & 16.7 & 0.001 \\
\hline & Taxanes alone & 288 & 30.9 & 174 & 23.6 & 114 & 58.5 & 0.001 & 111 & 27.7 & 141 & 31.1 & 33 & 47.1 & 3 & 50.0 & 0.001 \\
\hline & $\begin{array}{l}\text { Anthracyclines } \\
\text { alone }\end{array}$ & 14 & 1.5 & 13 & 1.8 & 1 & 0.5 & 0.323 & 6 & 1.5 & 8 & 1.8 & 0 & 0.0 & 0 & 0.0 & $0.71<$ \\
\hline & $\begin{array}{l}\text { Including } \\
\text { carboplatin }\end{array}$ & 29 & 3.1 & 25 & 3.4 & 4 & 2.0 & 0.486 & 12 & 3.0 & 14 & 3.1 & 2 & 2.9 & 1 & 16.7 & 0.29 \\
\hline & Other regimen & 25 & 2.7 & 16 & 2.2 & 9 & 4.6 & 0.078 & 10 & 2.5 & 8 & 1.8 & 6 & 8.6 & 1 & 16.7 & 0.002 \\
\hline \multicolumn{2}{|c|}{ Epirubicin dose $\geq 450 \mathrm{mg} / \mathrm{m}^{2}$} & 21 & 2.3 & 18 & 2.4 & 3 & 1.5 & 0.721 & 6 & 2.2 & 13 & 4.3 & 2 & 6.2 & 0 & 0.0 & $0.41 \epsilon$ \\
\hline \multicolumn{2}{|l|}{ Pertuzumab use } & 158 & 17.0 & 143 & 19.4 & 15 & 7.7 & 0.001 & 74 & 18.4 & 74 & 16.3 & 9 & 12.9 & 1 & 16.7 & 0.65 \\
\hline \multirow[t]{2}{*}{ Setting } & Adjuvant only & 584 & 62.7 & 434 & 59.0 & 150 & 76.9 & 0.001 & 235 & 58.6 & 291 & 64.1 & 54 & 77.1 & 4 & 66.7 & $0.02 \leftleftarrows$ \\
\hline & $\begin{array}{l}\text { Neoadjuvant } \\
\text { + adjuvant }\end{array}$ & 347 & 37.3 & 302 & 41.0 & 45 & 23.1 & 0.001 & 166 & 41.4 & 163 & 35.9 & 16 & 22.9 & 2 & 33.3 & $0.02=$ \\
\hline \multirow{2}{*}{$\begin{array}{l}\text { Radiotherapy } \\
\text { use }\end{array}$} & No & 242 & 26.0 & 179 & 24.3 & 63 & 32.3 & 0.027 & 106 & 26.4 & 111 & 24.4 & 24 & 34.3 & 1 & 16.7 & $0.33 \pi$ \\
\hline & Yes & 689 & 74.0 & 557 & 75.7 & 132 & 67.7 & 0.027 & 295 & 73.6 & 343 & 75.5 & 46 & 65.7 & 5 & 83.3 & $0.33 \pi$ \\
\hline \multirow[t]{3}{*}{$\begin{array}{l}\text { Endocrine } \\
\text { therapy }\end{array}$} & $\begin{array}{l}\text { No endocrine } \\
\text { therapy }\end{array}$ & 326 & 35.0 & 255 & 34.6 & 71 & 36.4 & 0.673 & 125 & 31.2 & 176 & 38.8 & 24 & 34.3 & 1 & 16.7 & 0.09 \\
\hline & Tamoxifen* & 379 & 40.7 & 338 & 45.9 & 41 & 21.0 & 0.001 & 212 & 52.9 & 149 & 32.8 & 14 & 20.0 & 4 & 66.7 & 0.001 \\
\hline & $\begin{array}{l}\text { Aromatase } \\
\text { inhibitor* }\end{array}$ & 226 & 24.3 & 143 & 19.4 & 83 & 42.6 & 0.001 & 64 & 16.0 & 129 & 28.4 & 32 & 45.7 & 1 & 16.7 & 0.001 \\
\hline \multirow{5}{*}{$\begin{array}{l}\text { Chemotherapy } \\
\text { cycles }\end{array}$} & Median & \multicolumn{2}{|l|}{6} & \multicolumn{2}{|l|}{6} & \multicolumn{2}{|l|}{4} & \multirow[t]{5}{*}{0.001} & \multicolumn{2}{|l|}{6} & \multicolumn{2}{|l|}{6} & 5 & & 5 & 0.001 & \\
\hline & IQR & $4-8$ & & $4-8$ & & $4-6$ & & & $4-8$ & & $4-8$ & & $4-6$ & & $4-6$ & & \\
\hline & Mean & 5.9 & & 6.2 & & 4.9 & & & 6.1 & & 5.9 & & 5.1 & & 4.7 & & \\
\hline & SD & 1.9 & & 1.8 & & 2.0 & & & 1.9 & & 1.9 & & 2.0 & & 2.7 & & \\
\hline & Range & $0-10$ & & $0-10$ & & $0-10$ & & & $0-1$ & & $0-1($ & & $1-8$ & & $0-8$ & & \\
\hline Epirubicin & Median & 360 & & 360 & & 360 & & 0.798 & 360 & & 360 & & 360 & & 360 & 0.852 & \\
\hline $\begin{array}{l}\text { dose } \\
\left(\mathrm{mg} / \mathrm{m}^{2}\right)^{\wedge}\end{array}$ & IQR & $300-$ & 360 & $300-$ & & $300-$ & 360 & & 300 & & 300 & 360 & 300 & -360 & $\begin{array}{l}360- \\
360\end{array}$ & & \\
\hline & Mean & 333.4 & & 333. & & 335.4 & & & 329. & & 336. & & 338 & & 360.0 & & \\
\hline & SD & 67.8 & & 67.7 & & 69.6 & & & 61.5 & & 73.4 & & 66.7 & & 0.0 & & \\
\hline & Range & $90-6$ & & $90-6$ & & $90-6$ & & & $90-$ & & $90-$ & & 180 & -600 & $\begin{array}{l}360- \\
360\end{array}$ & & \\
\hline Trastuzumab & Median & 18 & & 18 & & 18 & & 0.001 & 18 & & 18 & & 18 & & 18 & 0.193 & \\
\hline & IQR & $18-1$ & & $18-1$ & & $17-1$ & & & $18-$ & & $18-$ & & $17-$ & & $\begin{array}{l}14- \\
18\end{array}$ & & \\
\hline & Mean & 17.3 & & 17.5 & & 16.8 & & & 17.6 & & 17.3 & & 16. & & 15.0 & & \\
\hline & SD & 2.1 & & 1.7 & & 3.1 & & & 1.4 & & 2.2 & & 3.7 & & 5.6 & & \\
\hline & Range & $1-18$ & & $3-18$ & & $1-18$ & & & $3-1$ & & $1-1 \varepsilon$ & & $4-1$ & & $4-18$ & & \\
\hline
\end{tabular}


Table 4

Rates of cardiac events at any time following trastuzumab initiation in the overall population and according to age group and HFA-ICOS risk group.

\begin{tabular}{|c|c|c|c|c|c|c|c|c|c|c|c|c|c|c|c|c|c|c|}
\hline \multicolumn{2}{|l|}{ Cardiac events* } & \multicolumn{2}{|c|}{$\begin{array}{l}\text { Overall } \\
\mathrm{N}=931\end{array}$} & \multicolumn{2}{|c|}{ Age group } & & & \multicolumn{2}{|c|}{$p$ value } & \multicolumn{4}{|c|}{ HFA-ICOS risk category } & & & & & $\begin{array}{l}p \\
\text { val }\end{array}$ \\
\hline \multicolumn{2}{|l|}{$\begin{array}{l}<65 \text { years } \\
N=736\end{array}$} & \multicolumn{2}{|c|}{$\begin{array}{l}\geq 65 \text { years } \\
N=195\end{array}$} & & & \multicolumn{2}{|c|}{$\begin{array}{l}\text { Low } \\
\mathrm{N}=401\end{array}$} & \multicolumn{2}{|c|}{$\begin{array}{l}\text { Medium } \\
N=454\end{array}$} & \multicolumn{2}{|c|}{$\begin{array}{l}\text { High } \\
\mathrm{N}=70\end{array}$} & \multicolumn{2}{|c|}{$\begin{array}{l}\text { Very high } \\
N=6\end{array}$} & & & \multirow[b]{2}{*}{$\mathrm{N}$} & \multirow[b]{2}{*}{$\%$} & \multirow[b]{3}{*}{0.0} \\
\hline $\mathrm{N}$ & $\%$ & $\mathrm{~N}$ & $\%$ & & & $\mathrm{~N}$ & $\%$ & $\mathrm{~N}$ & $\%$ & $\mathrm{~N}$ & $\%$ & $\mathrm{~N}$ & $\%$ & & & & & \\
\hline \multicolumn{2}{|l|}{ Overall } & 155 & 16.6 & 116 & 15.8 & 39 & 20.0 & 0.161 & 56 & & 14.0 & 76 & 16.7 & 20 & 28.57 & 3 & 50.0 & \\
\hline \multicolumn{2}{|c|}{ LVEF decline $\geq 10 \%$} & 141 & 15.1 & 106 & 14.4 & 35 & 17.9 & 0.218 & 51 & & 12. & 70 & 15.42 & 17 & 24.3 & 3 & 50.0 & 0.0 \\
\hline \multicolumn{2}{|c|}{ LVEF decline below $50 \%$} & 55 & 5.9 & 43 & 5.8 & 12 & 6.1 & 0.865 & 18 & & 4.5 & 29 & 6.4 & 6 & 8.6 & 2 & 33.3 & 0.0 \\
\hline \multirow[t]{2}{*}{$\mathrm{CHF}$} & $\begin{array}{l}\text { NYHA } \\
\text { class II }\end{array}$ & 42 & 4.5 & 34 & 4.6 & 8 & 4.1 & 0.757 & 12 & & 3.0 & 24 & 5.3 & 4 & 5.7 & 2 & 33.3 & 0.0 \\
\hline & $\begin{array}{l}\text { NYHA } \\
\text { class III-IV }\end{array}$ & 5 & 0.5 & 3 & 0.4 & 2 & 1.0 & 0.294 & 0 & & 0.0 & 4 & 0.9 & 1 & 1.4 & 0 & 0.0 & 0.2 \\
\hline \multirow{3}{*}{$\begin{array}{l}\text { Trastuzumab } \\
\text { discontinuation } \\
\text { due to } \\
\text { cardiotoxicity }\end{array}$} & Overall & 35 & 3.8 & 26 & 3.5 & 9 & 4.6 & 0.040 & 9 & & 2.2 & 17 & 3.7 & 7 & 10.0 & 2 & 33.3 & 0.0 \\
\hline & Temporary & 23 & 2.5 & 18 & 2.4 & 5 & 2.6 & 0.999 & 5 & & 1.2 & 12 & 2.6 & 4 & 5.7 & 2 & 33.3 & 0.0 \\
\hline & Permanent & 12 & 1.3 & 8 & 1.1 & 4 & 2.0 & 0.289 & 4 & & 1.0 & 5 & 1.1 & 3 & 4.3 & 0 & 0.0 & 0.1 \\
\hline
\end{tabular}

Table 5

- Management of trastuzumab-related cardiac toxicity in the overall population and according to age group and HFA-ICOS risk group.

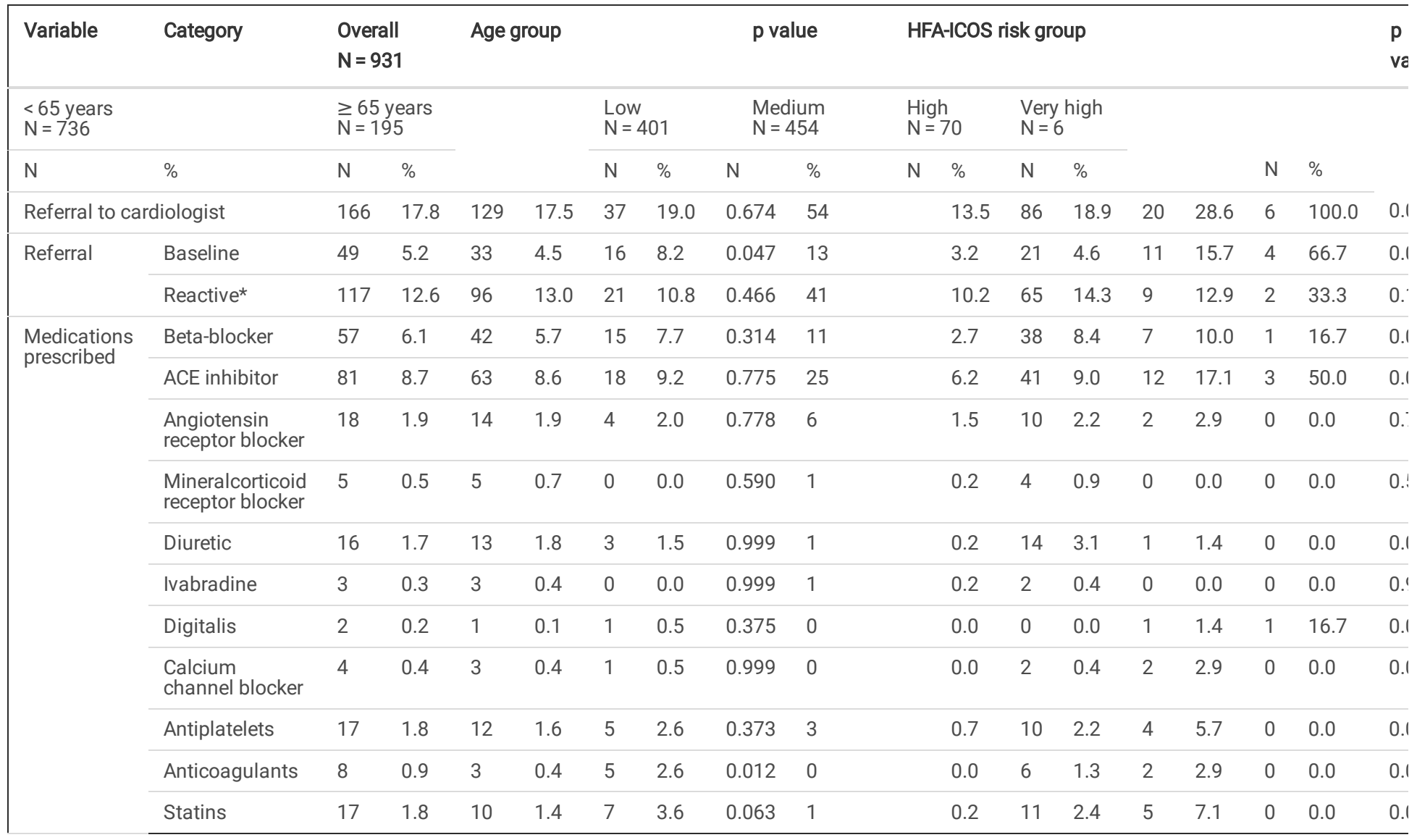

\section{Figures}




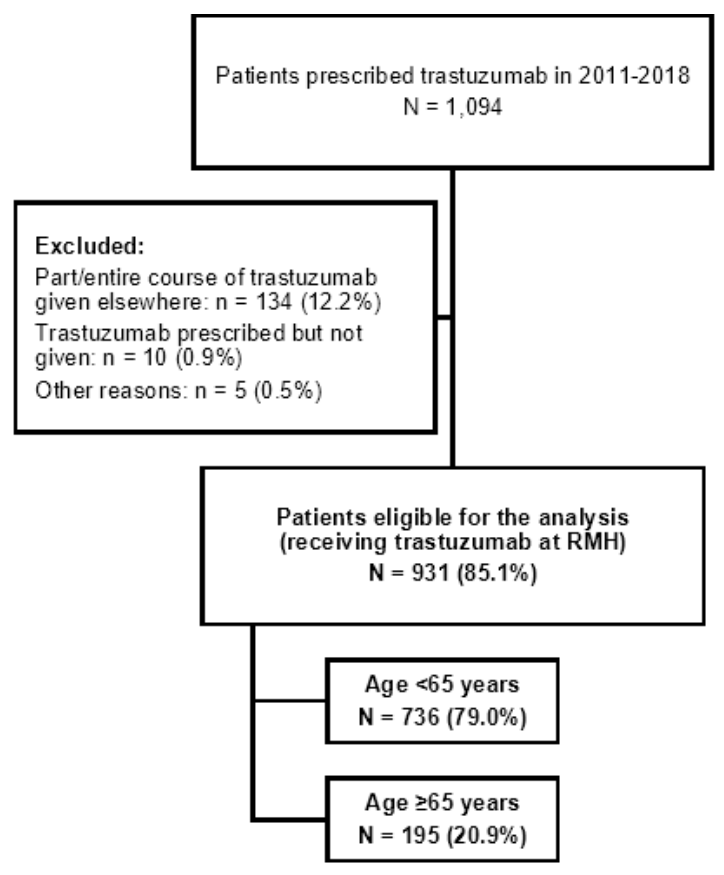

Figure 1

CONSORT diagram.

A.

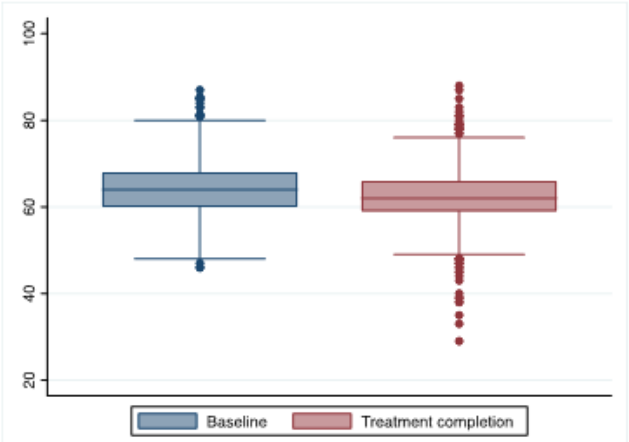

B.

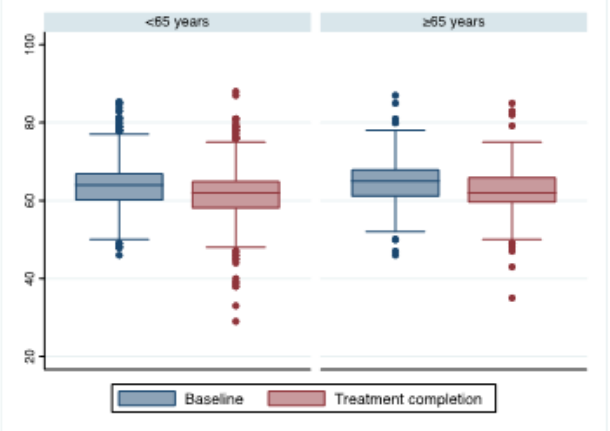

Figure 2

Left ventricular ejection fraction at baseline and upon trastuzumab completion in the overall population (A) and according to age group (B). 
A.

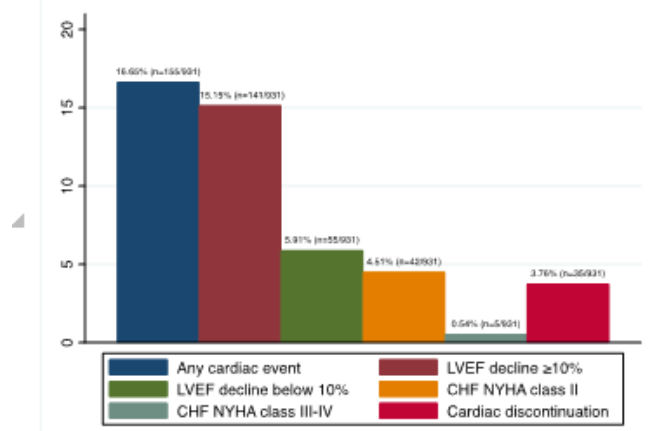

B.

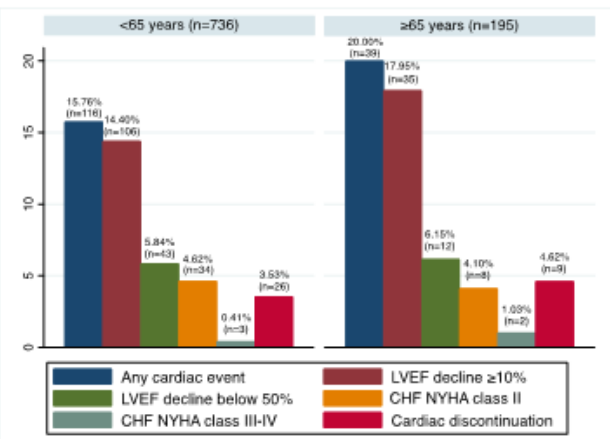

Abbreviations: LVEF: left ventricular ejection fraction; CHF: congestive heart failure; NYHA: New York Heart Association.

\section{Figure 3}

Rates of cardiac events at any time following trastuzumab initiation in the overall population (A) and according to age group (B).

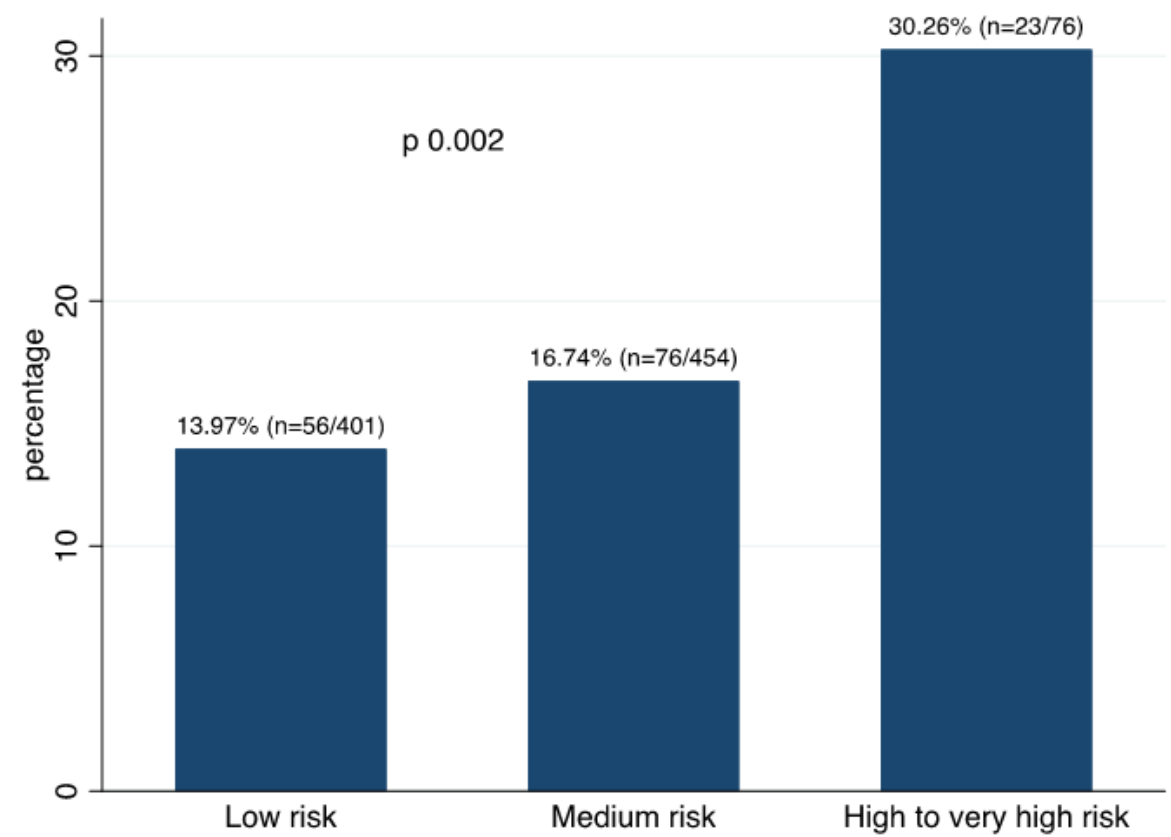

Figure 4

Rates of overall cardiac events by HFA-ICOS risk category. 